Reprinted with permission from: Journal of Plant Physiology. 1997. Vol. 151:603-609.

Published and copyrighted by: Gustav Fischer Verlag, Jena.

http://www.urbanfischer.de/journals

\title{
Polyamines, auxins and organogenesis in leafy spurge (Euphorbia esula L.) $)^{1}$
}

\author{
DAVID G. DAVIS
}

USDA, Agricultural Research Service, Biosciences Research Laboratory, P.O. Box 5674, State University Station Fargo, North Dakota 58105-5674 U.S.A.

\begin{abstract}
Summary:
Canaline and canavanine (inhibitors of polyamine biosynthesis) effects on root and shoot formation were determined in vitro for etiolated hypocotyl segments of leafy spurge (Euphorbia esula L.). Canaline (an ornithine analogue) at 10 to $100 \mu \mathrm{mol} / \mathrm{L}$ inhibited root and shoot formation. The simultaneous application of putrescine did not reverse canaline-induced inhibition of root formation. Ornithine, a precursor of putrescine via the ornithine decarboxylase pathway, unexpectedly inhibited root formation. Canavanine (an arginine analogue) at 30 to $100 \mu \mathrm{mol} / \mathrm{L}$ inhibited root and/or shoot formation. Inhibition of root formation by canavanine was not reversed by agmatine or putrescine, but was partially reversed by arginine. Indoleacetic acid (IAA) inhibited shoot formation, stimulated root formation, and partially reversed the inhibition of root formation by either canaline or canavanine. IAA generally increased endogenous levels of free polyamines in controls and canaline- or canavanine-treated tissues, but these changes were small and probably had little effect on organogenesis. IAA also partially reversed the inhibition of root (but not shoot) formation by arcaine and pentamidine, inhibitors of polyamine action in mammals. The roles of the polyamines in plants is not clear, but the polyamines may play only minor roles in root and shoot formation.
\end{abstract}

\section{Keywords:}

Putrescine, spermidine, spermine, IAA, indole-3-acetic acid, canaline, canavanine, ornithine, agmatine, arginine, arcaine, pentamidine.

${ }^{1}$ Received May 2, 1996 - Accepted February 7, 1997. 


\section{Abbreviations:}

Arcaine $=1,4$-diguanidinobutane sodium salt; canaline $=$ L- $\alpha$-amino- $y$ (aminooxy)-n-butyric acid dipicrate salt; canavanine $=2$, amino-4-(guanidinooxy)butyric acid sulfate salt; IAA = indole-3-acetic acid; pentamidine $=1,5$-bis[p-amidinophenoxy]-pentane bis[2-hydroxyethane]sulfonate salt; putrescine $=1$,4-diaminobutane; spermidine $=\mathrm{N}$-(3-aminopropyl)-1,4butanediamine; spermine $=\mathrm{N}, \mathrm{N}^{\prime}$ bis(3-aminopropyl)-1,4-butanediamine.

\section{Introduction}

Leafy spurge (Euphorbia esula L.) is an economically important perennial weed that requires alternative control methods for practical weed control (Watson, 1984; Lym and Messersmith, 1994). All parts of etiolated aseptically germinated leafy spurge seedlings are capable of regenerating new plants (Davis and Olson, 1993). Hypocotyl segments were used to determine the effects of auxins and cytokinins on the formation of visible roots and shoots, which occurs approximately seven to 10 days after excision, with almost no callus formation in control tissues (Davis and Olson, 1993). The polyamines putrescine, spermidine and spermine are involved in plant cell division and development (Slocum and Flores, 1991; Galston and Kaur-Sawhney, 1995). Therefore, the role of growth and development of roots and shoots in this plant species.

Two natural compounds, canaline and canavanine, inhibit putrescine biosynthesis via the ornithine decarboxylase (ODC) and arginine decarboxylase (ADC) pathways, respectively. Both canaline and canavanine are non-specific inhibitors of ODC and ADC (Altman and Bachrach, 1981) which regulate the two major pathways of putrescine biosynthesis in plants (Slocum and Flores, 1991). The inhibition of one pathway may not prevent polyamine biosynthesis, since the alternate pathway may still function if one pathway is inhibited. Also, arginine and ornithine are intercovertable, serving as potential alternate sources for the biosynthesis of putrescine and the polyamines.

The formation of roots and shoots in isolated hypocotyl segments of leafy spurge is inhibited by two inhibitors of putrescine biosynthesis, difluorylmethylornithine (DFMO) and difluoromethylarginine (DFMA) (Davis and Olson, 1994) that inhibit ODC and ADC, respectively (Slocum and Flores, 1991), and are reported to be specific suicide inhibitors of the two enzymes (McCann et al., 1987). A study similar to that of Davis and Olson (1994) was undertaken with canaline and canavanine, which are non-specific inhibitors of ADC and ODC, respectively. If similar results would be obtained, strong argument might be made for the involvement of putrescine and the polyamines in organ formation. If the results were totally different, evidence opposing polyamine involvement might result. Because canaline and canavanine are natural products, they might be more acceptable than DFMA or DFMO (or other synthesized compounds) to be considered for incorporation into an integrated weed control program. 
IAA partially overcomes DFMO- and DFMA-induced inhibition of root formation in leafy spurge isolated hypocotyl segments (Davis and Olson, 1994) so it was of interest to determine if IAA can overcome the inhibition of root formation by canaline and canavanine and to relate the results to polyamine levels. In addition, arcaine and pentamidine, two inhibitors of polyamine action (rather than polyamine biosynthesis) (Reynolds, 1990, 1992) were also tested for their effects on organogenesis and their interactions with IAA. Because of the large and consistent auxin responses on root formation in the previous report (Davis and Olson, 1994) the major emphasis in this report is on root formation, rather than shoot formation.

\section{Materials and methods}

Seeds collected from a field plot in southeastern North Dakota were surface sterilized with $70 \%$ ethanol followed by 20 minutes in $60 \%$ commercial bleach and three rinses in sterile water. The high concentration of bleach was required to eliminate a seed-borne contaminant. The seeds were germinated on sterile agar-water $(0.7 \% \mathrm{w} / \mathrm{v})$, in darkness at $30 / 20^{\circ} \mathrm{C}(12 / 12$ hours). After two weeks, the etiolated hypocotyls were cut into $1 \mathrm{~cm}$ segments (5-8 segments per hypocotyl) and placed onto $0.7 \%(\mathrm{w} / \mathrm{v})$ Phytagel (R) medium with B5 salts and vitamins (obtained from Sigma Chemicals, St. Louis, MO) and 2\% (w/v) sucrose (ultra pure, from ICN Biomedicals, Aurora, OH) (Gamborg et al., 1968). The tissue within $1 \mathrm{~cm}$ of the cotyledons and the root/shoot axis were discarded. Segments from individual hypocotyls were distributed randomly amongst the Petri dishes so that each dish received segments from several hypocotyls. Filter-sterilized aqueous solutions of canaline and canavanine were added to autoclaved media, mixed and poured into Petri plates prior to solidification. Acetone solutions of IAA were added to slightly cooled autoclaved medium at non-phytotoxic concentrations of acetone $(<0.1 \% \mathrm{v} / \mathrm{v}$; Davis et al., 1978). Where appropriate, acetone was also added to the control medium. Eight hypocotyl segments were used per $6 \mathrm{~cm}$ Petri dish, with 10 dishes (80 hypocotyl segments) per treatment.

Hypocotyl segments were weighed, frozen at $-20^{\circ} \mathrm{C}$, packed in solid $\mathrm{CO}_{2}$, sent by overnight express to the laboratory of Dr. S. Minocha, University of New Hampshire, and were analyzed as dansylated derivatives by high performance liquid chromatography (HPLC) (Minocha et al., 1990). The HPLC system consisted of a Perkin-Elmer Series 400 pump, fitted with a $10 \mu \mathrm{L}$ injection loop, a $25 \mathrm{~cm} \mathrm{C} \mathrm{C}_{18}$ reversed-phase column (Perkin-Elmer CRC18, particle size $3 \mu \mathrm{m}$ ), and a Perkin-Elmer fluorescence detector (excitation at $340 \mathrm{~nm}$, emission at $515 \mathrm{~nm}$ ). The dansylated polyamines were eluted using a linear gradient of acetonitrile and heptanesulfonate $(10 \mathrm{mM}$ in water, $\mathrm{pH} 3.4)$ at a flow rate of $2.5 \mathrm{~mL} /$ minute. Heptane diamine was used as an internal standard. A freeze-thaw method was used in the extraction of the leafy spurge hypocotyl segments (Minocha et al., 1994). Briefly, the hypocotyl segments were frozen and thawed three times in 5\% perchloric acid to accomplish the extraction. Similar results were obtained with samples of hypocotyl segments freeze-thawed three times and those ground for two minutes in a glass homogenizer.

IAA concentration was $1.1 \mu \mathrm{mol} / \mathrm{L}$ in all experiments. Controls were grown on the same medium with no exogenous growth regulators. The Petri dishes were wrapped with

Page 3 of 11 
Parafilm ${ }^{\circledR}$ and aluminum foil and incubated in darkness at $26^{\circ} \mathrm{C}$. Roots and shoots were observed at seven and (usually) 28 days by the use of a dissecting microscope, and they were counted when they were clearly recognizable as distinct organs (1-2 mm long). Callus formation on hypocotyl segments treated with IAA, canaline or canavanine was negligible.

Experiments were repeated two or more times. Triplicate samples within a given experiment were used to determine polyamine concentrations. Comparisons were made to controls or between treatments using two-sample-t-tests (Statistix, NH Analytical Software, St. Paul, MN).

\section{Results}

\section{Canaline and canavanine effects on organogenesis and free poly- amine levels}

Canaline and canavanine inhibited both root and shoot formation in isolated hypocotyl segments of leafy spurge at concentrations greater than 10 and $30 \mu \mathrm{mol} / \mathrm{L}$, respectively (Fig. 1). In control hypocotyl segments, endogenous free putrescine declined by day two (Fig. 2), leveling off at approximately $150 \mathrm{nmol} / \mathrm{g}$ (fresh mass) for the next seven days followed by a slow decline to about $100 \mathrm{nmol} / \mathrm{g}$ (fresh mass) by day 16. Sixty micromoles per liter of either canaline or canavanine reduced the levels of putrescine below that of the controls, within two days, approaching zero by 16 days of culture.

Unlike free putrescine, endogenous levels of free spermidine in control tissues increased nearly four fold by 16 days of culture (Fig. 2). Canaline or canavanine alone (60 $\mu \mathrm{mol} / \mathrm{L}$ ) inhibited the level of free spermidine about equally, to just over $50 \%$ of the concentration in the controls at day 16.

Free spermine levels were much lower than either free putrescine or free spermidine, increasing in controls from nearly zero to approximately $40 \mathrm{nmol} / \mathrm{g}$ (fresh mass) (Fig. 2). Neither canaline nor canavanine had a significant effect on the free spermine concentration up to 16 days of culture.

\section{Effects of exogenous putrescine or its precursors on root formation}

Exogenous putrescine, or one of its precursors, was added simultaneously with either canaline or canavanine to test whether they might overcome the canaline- or canavanine-induced inhibition

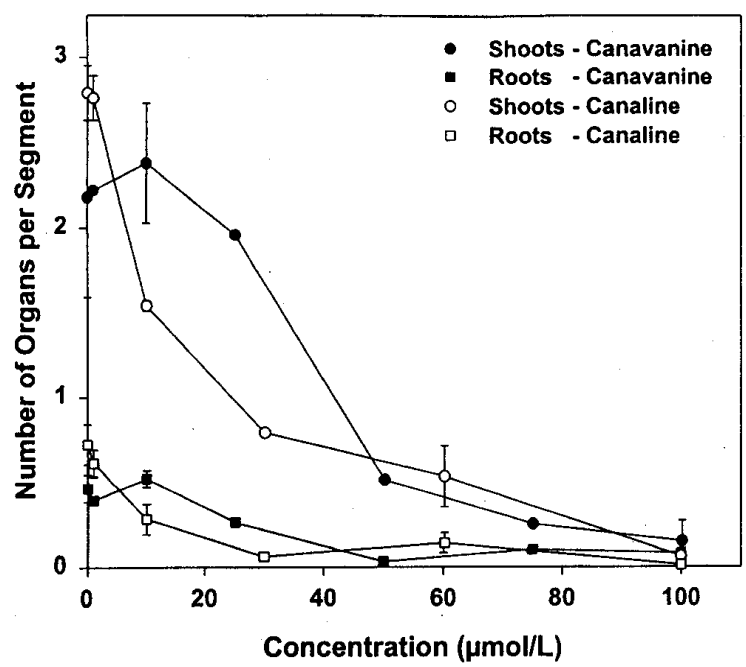

Fig. 1: Effects of canaline (open symbols) and canavanine (closed symbols) on root and shoot formation in leafy spurge hypocotyl segments. Vertical bars are \pm standard errors. 
of root formation. Exogenous putrescine caused slight increases or decreases in root numbers, while canaline (Fig. 3) or canavanine (Fig. 4) inhibited root formation. Putrescine at $150 \mu \mathrm{mol} / \mathrm{L}$ or $1000 \mu \mathrm{mol} / \mathrm{L}$ combined with canaline increased root num6ers over canaline alone, but only to 52 and $27 \%$ of the controls, respectively (Fig. 3A). Unexpectedly, ornithine by itself inhibited root formation (Fig. 3B), and ornithine+canaline resulted in relatively little change from canaline or ornithine alone with significantly fewer roots than controls $(\mathrm{p}<0.05)$.

Putrescine+canavanine increased the number of roots slightly above that of canavanine alone, but to less than $40 \%$ of the control levels (Fig. 4A). Agmatine (an intermediate between arginine and putrescine) at $1000 \mu \mathrm{mol} / \mathrm{L}$ and agmatine+canavanine increased root formation slightly above that of canavanine alone, but still only to $35 \%$ of the control values (Fig. 4 B). Arginine at $100 \mu \mathrm{mol} / \mathrm{L}$ or $1000 \mu \mathrm{mol} / \mathrm{L}$ had no significant effect on roots numbers (Fig. $4 \mathrm{C}$ ), and only $1000 \mu \mathrm{mol} / \mathrm{L}$ arginine partially overcame the effects of canavanine alone, but to only $67 \%$ of the controls (not significantly different from the controls at $\mathrm{p}<$ $0.05)$.

Thus it appears that, when added alone, putrescine and/or precursors of putrescine biosynthesis have only a small effect on root formation in leafy spurge hypocotyl segments. Only arginine appeared to partially overcome the canavanine-induced inhibition of root formation.

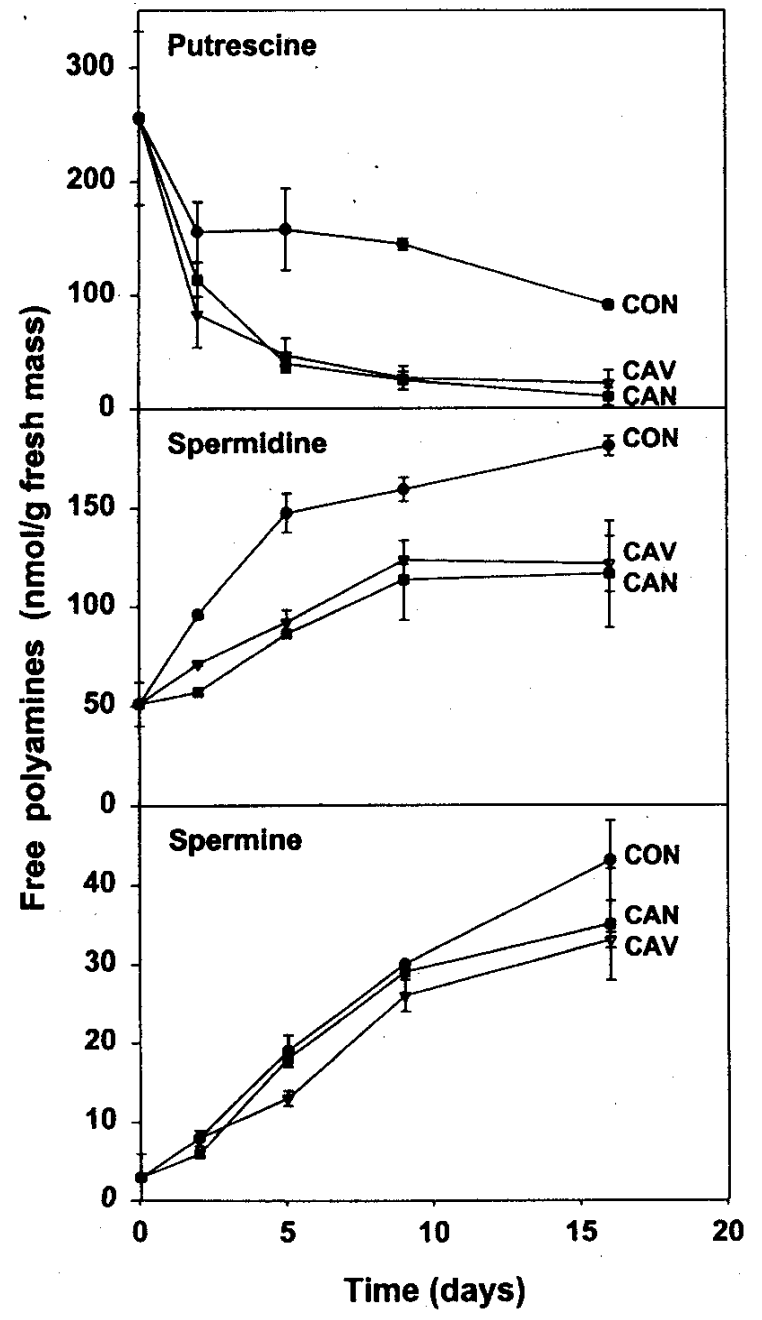

Fig. 2: Effects of $60 \mu \mathrm{mol} / \mathrm{L}$ canaline and canavanine over time on the concentration of free polyamines in leafy spurge hypocotyl segments. 


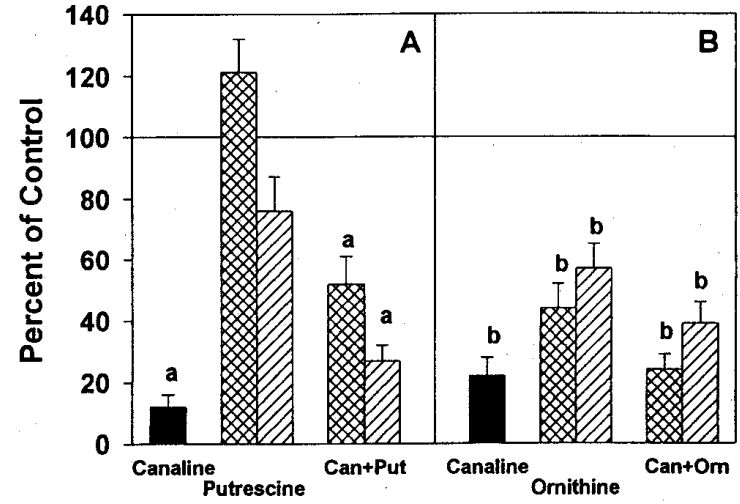

Fig. 3: Effects of canaline, putrescine, ornithine and combinations of the two on root formation in leafy spurge hypocotyl segments. Values are numbers of roots per hypocotyl segment expressed as a percent of the controls. Representative experiments with putrescine $(A)$ and ornithine $(B)$, where controls averaged 1.02 (A) and 1.12 (B) roots/hypocotyl segment. Canaline was at 50 $\mu \mathrm{mol} / \mathrm{L}$, putrescine at 150 and $1000 \mu \mathrm{mol} / \mathrm{L}$, and ornithine at 100 and $1000 \mu \mathrm{mol} / \mathrm{L} . \mathrm{a}, \mathrm{b}$ $=$ values differ from controls at $\mathbf{p}<0.01$ or 0.05 , respectively.

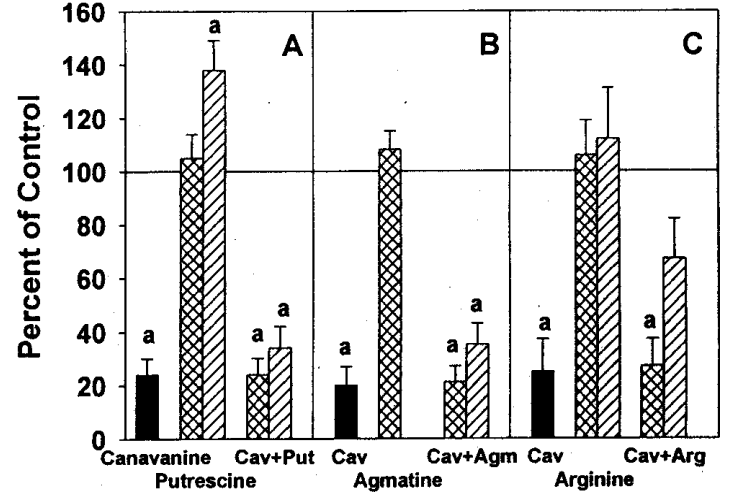

Fig. 4: Effects of canavanine, putrescine, agmatine, arginine and combinations of canavanine with the other three on root formation in leafy spurge hypocotyl segments. Values are numbers of roots per hypocotyl segment expressed as a percent of the controls. Representative experiments with putrescine (A), agmatine (B) and arginine (C), where controls averaged $1.01(\mathrm{~A}), 0.71$ (B) and 0.52 (C) roots/ hypocotyl segment. Canavanine was at $60 \mu \mathrm{mol} / \mathrm{L}$ and putrescine, agmatine or arginine were at 100 and 1000 $\mu \mathrm{mol} / \mathrm{L} . \mathrm{a}=$ values differ from controls at $\mathbf{p}<\mathbf{0 . 0 1}$.

\section{Effect of IAA on canaline- and canavanine-induced inhibition of root formation}

In previous work (Davis and Olson, 1994) IAA was found to partially overcome the inhibition of root formation by the combination of DFMO+DFMA, the two previously described specific inhibitors of ODC and ADC, respectively. It was decided to establish whether a similar response would be observed with canaline and canavanine. The difference in the approach was that a combination of both DFMO and DFMA were needed to completely inhibit both root and shoot formation, whereas either canaline alone or canavanine alone was effective.

Shoot formation was inhibited about equally by $1.1 \mu$ IAA, $50 \mu \mathrm{M}$ canaline (Fig. 5) or $60 \mu \mathrm{M}$ canavanine (Fig. 6). IAA had no significant effect on canaline- or canavanineinduced inhibition of shoot formation.

Root formation was also inhibited, strongly by either canaline (Fig. 5) or canavanine (Fig. 6), but, in contrast to the effects on shoot formation, IAA greatly stimulated root formation. The combination of IAA+canaline increased root numbers to greater than controls, while IAA+canavanine increased root numbers to just slightly greater than control levels. Neither combination increased root numbers to the levels of IAA alone. The difference in response is undoubtedly due to the fact that canavanine is somewhat more in- 
hibitory to root formation than is canaline and was at a slightly higher concentration (Fig. 1).

\section{IAA-induced changes in free polyamine levels}

Putrescine levels decreased with time in all tissues, including controls (Fig. 7). IAA-treated tissues contained approximately double the putrescine level of the untreated tissues. Canaline and canavanine by themselves reduced putrescine levels slightly below the controls. IAA increased putrescine levels in the presence or absence of canaline, but IAA+canavanine resulted in lower putrescine levels than either canaline or canavanine alone.

Spermidine levels in IAA-treated tissues were significantly higher $(p<0.05)$ than controls at 3 days of culture but not at 10 days (Fig. 7). At 3 days IAA+canaline induced higher spermidine levels than canaline alone. All other treatments (canaline alone, canavanine alone, and IAA+canavanine) resulted in lower spermidine levels than the controls.

Spermine levels were low initially, increasing with time in controls and in all treated tissues to approximately 25 to 40 $\mathrm{nmol} / \mathrm{g}$ (fresh mass) by 10 days (Fig. 7).

IAA had little or no effect on spermine levels, with or without either canaline or canavanine (Fig. 7).

\section{Effects of combining IAA with arcaine and pentamidine, inhibitors of polyamine action}

Arcaine and pentamidine inhibited both shoot and root formation in leafy spurge hypocotyl segments (Table 1). Shoot formation was decreased further by IAA applied simultaneously with either arcaine or pentamidine. Root formation was increased significantly above control levels by IAA+arcaine or IAA+pentamidine, but not to the level of IAA alone. 
Table 1. Effects of arcaine, pentamidine and IAA on organogenesis in isolated hypocotyl segments of leafy spurge.

\begin{tabular}{lccc}
\hline & $\begin{array}{c}\text { Concentration } \\
\text { Treatment }\end{array}$ & \multicolumn{2}{c}{ Number per segment of } \\
\cline { 3 - 4 } & - & 3.3 & Shoots \\
\hline Controls & 100 & $1.3^{\mathrm{a}}$ & 1.2 \\
Arcaine & 1 & $0.6^{\mathrm{a}}$ & $0.5^{\mathrm{a}}$ \\
IAA & $100+1$ & $0.3^{\mathrm{a}}$ & $3.3^{\mathrm{a}}$ \\
Arcaine + IAA & - & 2.6 & $2.4^{\mathrm{a}}$ \\
Controls & 10 & $0.2^{\mathrm{a}}$ & 1.2 \\
Pentamidine & 1 & $0.2^{\mathrm{a}}$ & $0.2^{\mathrm{a}}$ \\
IAA & $10+1$ & $0.01^{\mathrm{a}}$ & $8.3^{\mathrm{a}}$ \\
Pentamidine + IAA & & & $2.3^{\mathrm{a}}$ \\
\hline
\end{tabular}

Differs from controls $(\mathrm{p}<0.001)$.

\section{Discussion}

Canavanine is a non-protein, amino acid analogue of arginine found in many legumes that inhibits the growth of isolated roots of soybean, bean and corn (Weaks and Hunt, 1974). Canavanine inhibits the incorporation of arginine into putrescine in tuber slices of Helianthus tuberosus (L.) (Bagni et al., 1981) and inhibits arginine decarboxylase (ADC) (Mengoli et al., 1989). Canaline and canavanine are approximately ten times as inhibitory as DFMO or DFMA in leafy spurge hypocotyl segments; being effective at ca. $50 \mu \mathrm{mol} / \mathrm{L}$ (this report) compared to ca. $500 \mu \mathrm{mol} / \mathrm{L}$ for DFMO or DFMA (Davis and Olson, 1994). In plantlets of a Cichorium intybus (L.) hybrid cultured in vitro, Helleboid et al., 1995 found somatic embryogenesis inhibited by DFMA, the specific inhibitor of ADC. DFMA also reduced putrescine and spermine concentrations in the hybrid, but the inhibition by DFMA was reversed by $5 \mathrm{mmol} / \mathrm{L}$ putrescine. In the report by Davis and Olson (1994), exogenous putrescine partially reversed DFMA-induced inhibition of root formation; but in the present work $1000 \mu \mathrm{M}$ putrescine (at a 17:1 ratio of putrescine:canavanine) did not overcome the inhibition by either canaline or canavanine.

Canavanine is a non-specific inhibitor of ADC since it also inhibits the induction of nitrate reductase (Srivastava, 1975), RNA synthesis in Helianthus tuberosus (L.) (Bagni et al., 1981), and RNA synthesis as well as subsequent protein synthesis in Chlamydomonas reinhardi [sic] (McMahon and Langstroth, 1972). Canavanine can be substituted for arginine in proteins (McMahon and Langstroth, 1972) and is hydrolyzed to canaline and urea (Rosenthal, 1977). The inhibition of organogenesis in leafy spurge hypocotyl segments by canavanine, observed in this laboratory, might result from such a substitution of canavanine in proteins required for cell division and/or development, and therefore be unrelated to its effect on polyamine biosynthesis.

Because putrescine is synthesized in many plants via two pathways, it was presumed that treatment with canaline or canavanine alone might not greatly affect the endogenous concentration of putrescine. Therefore, the concentration of putrescine in the tissues 
might remain relatively constant even in the presence of either inhibitor. However, in leafy spurge hypocotyl segments, both canaline and canavanine reduced free putrescine and free spermidine concentrations compared to controls, supporting the concept that organogenesis in leafy spurge hypocotyl segments may be linked to changes in polyamine levels.

Arginine overcame canavanine inhibition of root formation in mung bean hypocotyls (Altman and Bachrach, 1981) and partially reversed the inhibition of root elongation in Zea mays (L.), but not in Phaseolis vulgaris (L.) or Glycine Max (L.) Merr. (Weaks and Hunt, 1974), where the effects of canavanine may be more complex than a simple competition with arginine and ADC. In leafy spurge hypocotyl segments, arginine only partially overcame the canavanine inhibition of root formation. Canavanine may replace arginine in proteins other than ADC that are required for organ initiation and/or development, resulting in reduced root and shoot formation.

Canaline- and canavanine-induced inhibition of root formation on mungbean cuttings (Altman and Bachrach, 1981) was almost totally reversed by the addition of arginine and ornithine (the respective naturally-occurring analogs of canavanine and canaline) and was partially reversed by the synthetic auxin indolebutyric acetic acid. In leafy spurge hypocotyl segments, ornithine, in the absence of exogenous auxin, inhibited organogenesis and did not overcome the inhibition of organogenesis by canaline alone. This appears to argue against putrescine biosynthesis as a regulator of organogenesis.

IAA is a strong promotor of root formation and inhibited shoot formation in leafy spurge hypocotyl segments. IAA increases polyamine content in tuber slices of Helianthus tuberosus (L.) (Cocucci and Bagni, 1968). Because IAA stimulated putrescine levels in leafy spurge hypocotyl segments and also overcame much of the canaline- or canavanine-induced inhibition of root formation, there is argument in favor of putrescine as a regulator of root and/or shoot formation. However, putrescine levels in controls declined in the leafy spurge hypocotyl segments during the first few days of culture (the period in which most roots and shoots are formed; Davis and Olson, 1993, 1994), so or- 
ganogenesis in the control tissues appears to be inversely, rather than directly, related to putrescine levels in the tissues. The changes in concentration of putrescine, spermidine or spermine induced by IAA may not be sufficiently large to influence significantly the regulation of root or shoot formation.

Hausman et al. (1994) concluded that the degradative pathway of putrescine to $\Delta^{\prime}$-pyrroline is the important regulatory pathway in which polyamines are involved in root formation in poplar. Perhaps the decline in putrescine content and the subsequent effect on organ formation in leafy spurge is analogous to that in poplar.

There are no published reports of the effects of either arcaine or pentamidine on plants. In mammals, both compounds appear to be involved in polyamine action, rather than in polyamine biosynthesis (Reynolds, 1990, 1992). Arcaine, also known as diamidino-putrescine, is an N-substituted derivative of arginine that inhibits nitric oxide synthase and increases the influx of cytosolic calcium in rats (Kabuto et al., 1995). Arcaine appears to interact with polyamines via an N-methyl-D-aspartate (NMDA) glutamate receptor in rat brains; perhaps outcompeting the polyamines for one of two sites of NMDA action. However, the exact interaction of arcaine with the polyamines remains elusive.

Pentamidine also antagonizes the NMDA receptor. It does not appear to compete with the polyamine site, but interacts somehow with zinc and spermidine (Reynolds, 1992). The inhibition of organogenesis with arcaine (at $100 \mu \mathrm{mol} / \mathrm{L}$ ) and especially, pentamidine (at $10 \mu \mathrm{mol} / \mathrm{L}$ ) give support to the possibility of polyamine involvement in root and shoot development. IAA counteracts much of the inhibition of root formation by both compounds indicating that auxin is the primary inducer of root formation. The results of experiments with the various inhibitors of polyamines described in this report lead to somewhat conflicting conclusions. Polyamines may be involved in, but may not be crucial to root and shoot formation in leafy spurge hypocotyl segments. Their exact role(s) remain unclear.

\section{Acknowledgements}

The author wishes to thank Cheryl Kimberlin and Kristi Biewer Breiland for excellent technical assistance, and especially Dr. Subhash Minocha, University of New Hampshire for polyamine analyses.

\section{References}

Altman, A. and U. Bachrach. 1981. Involvement of polyamines in plant growth and senescence. Advances in Polyamine Research 3, 365-375.

Bagni, N., P. Torrigiani, and P. Barbieri. 1981 Effect of various inhibitors of polyamine synthesis on the growth of Helianthus tuberosus. Medical Biology 59, 403-409.

Cocucci, S. and N. Bagni. 1968. Polyamine-induced activation of protein synthesis in ribosomal preparation from Helianthus tuberosus tissue. Life Sciences 7:113-120.

Davis, D. G. and P. A. Olson. 1993. Organogenesis in leafy spurge (Euphorbia esula L.). In Vitro Cell. Dev. Biol. 29P:97-101.

Page 10 of 11 
Davis, D. G. and P. A. Olson. 1994. Effects of putrescine and inhibitors of putrescine biosynthesis on organogenesis in Euphorbia esula L. In Vitro Cell. Dev. Biol. 30P:124-130.

Davis, D. G., W. Wergin, and K. E. Dusbabek. 1978. Effects of organic solvents on growth and ultrastructure of plant cell suspensions. Pestic. Biochem. Physiol. 8:84-97.

Galston, A. W and R. Kaur-Sawhney. 1995. Polyamines as endogenous growth regulators. In: Davies, - P. J. (ed.): Plant Hormones, pp. 158-178. Kluwer Academic Press, Dortrecht.

Gamborg, O. L., R. A. Miller, and K. Ojima. 1968. Nutrient requirements of suspension cultures of soybean root cells. Exp. Cell Res. 50:148-151.

Hausman, J. E., C. Kevers, and T. Gaspar. 1994. Involvement of putrescine in the inductive rooting phase of poplar shoots raised in vitro. Physiol. Plant. 92:201-206.

Helleboid, S., J. P. Couillerot, J. L. Hilbert, and J. Vasseur. 1995. Inhibition of direct somatic embryogenesis by $\alpha$-difluoromethylarginine in a Cichorium hybrid: Effects on polyamine content and protein patterns. Planta 196:571-576.

Kabuto, H., I. Yokoi, H. Habu, H. Asahara, and A. Mori: Inhibitory effect of arcaine on nitric oxide synthase in the rat brain. Neuro Report 6:554-556.

Lym, R. G. and C. G. Messersmith. 1994. Leafy spurge (Euphorbia esula) control, forage production and economic return with fall-applied herbicides. Weed Technology 8:824-829.

McCann, P. P, A. E. Pegg, and A. Sjoerdsma (eds.). 1987. Inhibition of Polyamine Metabolism. Biological Significance and Basis for New Therapies, 371 pp. Academic Press, Inc., Orlando, FL, USA.

McMahon, D. and P. Langstroth. 1972. The effects of canavanine and of arginine starvation on macromolecular synthesis in Chlamydomonas reinhardi. J. General Microbiol. 73:239-250.

Mengoli, M., N. Bagni, G. Luccarini, V. Nutto-Ronchi, and D. Serafini-Fracassini. 1989. Daucus carota cell cultures: Polyamines and effect of polyamine biosynthesis inhibitors in the preembryogenic phase and different embryo stages. J. Plant Physiol. 134:389-394.

Minocha, S. C., R. Minocha, and C. A. Robie. 1990. High performance liquid chromatographic method for the determination of dansylpolyamines. J. Chromatogr. 511:177-183.

Minocha, R., W. C. Shortle, S. L. Long, and S. C. Minocha. 1994. A rapid and reliable procedure for extraction of cellular polyamines and inorganic ions from plant tissues. J. Plant Growth Regul. 13:187-193.

Reynolds, I. J. 1990. Arcaine is a competitive antagonist of the polyamine site on the NMDA receptor. Eur. J. Pharmacol. 177:215-216.

Reynolds, I. J. 1992. Interactions between zinc and spermidine on the N-methyl-D-aspartate receptor complex: Clues to the mechanism of action of 1,10-bis(guanidino)decane and pentamidine. J. Pharmacol \& Exper. Therapeutics 263:632-638.

Rosenthal, G. A. 1977. The biological effects and mode of action of L-canavanine, a structural analogue of L-arginine. Quart. Rev. Biol. 52:155-178.

Slocum, R. D. and H. E. Flores, (eds.). 1991. Biochemistry and Physiology of Polyamines in Plants, pp. 2340. CRC Press, Boca Raton, FL, USA.

Srivastava, H. S. 1975. Inhibition of nitrate reductase induction by canavanine in maize roots. Phytochemistry 14:1951-1953.

Watson, A. K. (ed.). 1985 Leafy Spurge. Monograph no. 3, 104 pp. Weed Science Society of America. Champaign, IL, USA.

Weaks, T. E. and G. E. Hunt. 1974. The effects of canavanine on the growth of isolated roots of four plant species. Bot. Gaz. 135:45-49. 